\title{
STRONG WEAK INTERACTIONS
}

\author{
R. PHILIPPE* \\ Randall Laboratory of Physics, University of Michigan, Ann Arbor, MI 48109, USA
}

Received 19 June 1984

\begin{abstract}
We study the intriguing and attractive possibility that the standard model Higgs particle is very heavy or does not exist at all and that, as a consequence, a spectrum of vector boson bound states emerges.
\end{abstract}

Although the standard model is extremely successful in describing low-energy physics, the question of symmetry breaking is still completely open. The renormalizability of the theory technically requires the presence of a scalar Higgs particle (its mass is a free parameter of the model). But the Higgs also causes very serious theoretical problems, one of the most severe being the cosmological constant [1], so that its very existence as a physical particle is undoubtedly questionable.

In this article we want to elaborate on the possibility and consequences of a heavy Higgs particle [2] and, going only one step further, of the complete absence of such a particle. One of its most striking consequences is a possible violation of unitarity for scattering of longitudinally polarized weak vector bosons off each other in the Born approximation (dominated by Higgs exchanges) [3,4]. This, however, does not lead to an absolute upper bound on the Higgs mass. The situation is far more complicated.

Let us concentrate on $\mathrm{W}_{\ell}^{+} \mathrm{W}_{\ell}^{-}$scattering. Seven diagrams contribute in lowest order: a contact graph and $\gamma-, \mathrm{Z}$ - and H-exchanges in the $s$ - and $t$-channel. The corresponding contributions to the amplitude proportional to $\left(s / m_{\mathrm{W}}^{2}\right)^{2}$ and $s / m_{\mathrm{W}}^{2}$ cancel each other in the high-energy limit. The remaining terms originating from the contact and $\gamma$ - and Z-diagrams are of order $\alpha_{\text {weak }}$, while exchanging H's (see fig. 1) leads to

$$
A^{0}\left(\mathrm{~W}_{\ell}^{+} \mathrm{W}_{\ell}^{-} \rightarrow \mathrm{W}_{\ell}^{+} \mathrm{W}_{\ell}^{-}\right)=-\frac{1}{4} \frac{m_{\mathrm{H}}^{2}}{m_{\mathrm{W}}^{2}} g^{2}\left(\frac{s}{s-m_{\mathrm{H}}^{2}}+\frac{t}{t-m_{\mathrm{H}}^{2}}\right)
$$

* Supported in part by the US Department of Energy. 
which is proportional to $m_{\mathrm{H}}^{2}$. Expression (1) therefore dominates the amplitude for high energies in case of a heavy Higgs particle.

The corresponding s-partial wave amplitude for $s$ and $m_{\mathrm{H}}^{2} \gg m_{\mathrm{W}}^{2}$ is given by

$$
A_{s}^{0}=\frac{1}{64 \pi} \frac{m_{\mathrm{H}}^{2}}{m_{\mathrm{W}}^{2}} g^{2}\left\{\frac{1}{1-\hat{s}}+\frac{1}{\hat{s}} \ln (1+\hat{s})-2\right\},
$$

with $\hat{s}=s / m_{\mathrm{H}}^{2}$. It is immediately clear that unitarity is violated for a sufficiently heavy Higgs. The p-and d-wave projections are however well-behaved:

$$
\begin{aligned}
& A_{\mathrm{p}}^{0}=\frac{1}{64 \pi} \frac{m_{\mathrm{H}}^{2}}{m_{\mathrm{W}}^{2} \hat{s}} g^{2}\left\{\left(1+\frac{2}{\hat{s}}\right) \ln (1+\hat{s})-2\right\}, \\
& A_{\mathrm{d}}^{0}=\frac{3}{32 \pi} \frac{m_{\mathrm{H}}^{2}}{m_{\mathrm{W}}^{2} \hat{s}} g^{2}\left\{-\frac{1}{\hat{s}}+\left(\frac{1}{6}+\frac{1}{\hat{s}}+\frac{1}{\hat{s}^{2}}\right) \ln (1+\hat{s})-\frac{1}{2}\right\} .
\end{aligned}
$$

They are proportional to $1 / s$. From (2) we derive the critical Higgs mass

$$
m_{\mathrm{H}}^{\mathrm{c}}=4 \sqrt{2 \pi} m_{\mathrm{W}} g^{-1},
$$

which is $m_{\mathrm{H}}^{\mathrm{c}}=1.234 \mathrm{TeV}$. Examination of other vector boson-vector boson scattering amplitudes leads to similar critical values (e.g. $Z_{\ell} Z_{\ell} \rightarrow Z_{\ell} Z_{\ell}$ yields $m_{\mathrm{H}}^{c}=1.425$ $\mathrm{TeV}$ ). The meaning of this particular Higgs mass is the following: if the Higgs particle is heavier than value (5), then unitarity is violated at the tree level, so that, because gauge theories do satisfy unitarity, higher-order effects must become very important and weak interactions among massive vector bosons become strong. There are thus two possibilities:

(i) $m_{\mathrm{H}}<m_{\mathrm{H}}^{\mathrm{c}}$ : in this case the weak interactions remain weak at all energies.

(ii) $m_{\mathrm{H}}>m_{\mathrm{H}}^{\mathrm{c}}$ : this implies that perturbation theory breaks down at some finite energy.

Let us focus on the latter case. Here to every critical Higgs mass $m_{\mathrm{H}}^{\mathrm{c}}$ corresponds a critical energy $s_{\mathrm{c}}$, which separates the weak from the strong regime. It is, however,

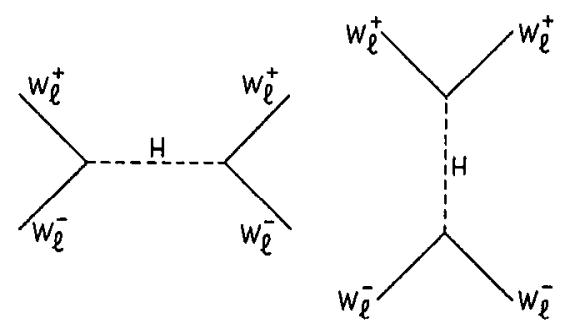

Fig. 1. Exchange of Higgs in $s$ - and $t$-channel for $\mathrm{W}_{\ell}^{+} \mathrm{W}_{\ell}^{-}$scattering. 
extremely difficult to determine $s_{\mathrm{c}}$ as a function of $m_{\mathrm{H}}^{\mathrm{c}}$. The reason is the divergence of the first term of eq. (2) at the Higgs pole. The width $\Gamma_{\mathrm{H}}$ of the Higgs particle (see e.g. [5]) has to be taken into account. The problem is that $\Gamma_{\mathrm{H}}$ is extremely large for a heavy Higgs $\left(\Gamma_{\mathrm{H}}-m_{\mathrm{H}}^{3}\right) . \Gamma_{\mathrm{H}}$ already equals $m_{\mathrm{H}}$ for $m_{\mathrm{H}} \approx 1.4 \mathrm{TcV}$. This too makes a physical Higgs particle suspicious. Naively replacing

$$
m_{\mathrm{H}} \rightarrow\left(1-\frac{3 i}{256 \pi} g^{2} \frac{m_{\mathrm{H}}^{2}}{m_{\mathrm{W}}^{2}}\right) m_{\mathrm{H}}
$$

in the s-wave (2) leads to $s_{\mathrm{c}}=m_{\mathrm{H}}^{2}$ [4] or even $s_{\mathrm{c}}=m_{\mathrm{H}}^{2}+2 \Gamma_{\mathrm{H}}^{2}$ [6]. This implies that the larger the Higgs mass the later Born unitarity is violated. Exactly the opposite behavior is expected: the heavier the Higgs the sooner weak interaction perturbation theory breaks down. This only means that replacement (6) is no longer reliable (it is however remarkable that substituting (6) only in $\left(s-m_{\mathrm{H}}^{2}\right)$ of (2) yields the expected kind of behavior). But it is nevertheless highly unlikely that, even for a superheavy Higgs particle, $\sqrt{s_{\mathrm{c}}}$ can become very small, say of the order of $750 \mathrm{GeV}$. This is an immediate consequence of the screening theorem [7]. It will be extremely difficult to observe any direct strong interaction effects at relatively low energies, except perhaps in the process $\mathrm{e}^{+} \mathrm{e}^{-} \rightarrow \mathrm{W}^{+} \mathrm{W}^{-}[8]$.

The various longitudinally polarized vector boson-vector boson scattering processes contribute to an isoscalar and isotensor eigenchannel. Their s-wave projections for $s$ and $m_{\mathrm{H}}^{2} \gg m_{\mathrm{W}}^{2}$ are given by

$$
\begin{aligned}
& A_{\mathrm{s}}^{0}(I=0)=\frac{3}{128 \pi} \frac{m_{\mathrm{H}}^{2}}{m_{\mathrm{W}}^{2}} g^{2}\left\{\frac{1}{1-\hat{s}}+\frac{2}{3 \hat{s}} \ln (1+\hat{s})-\frac{\hat{s}}{3}\right\}, \\
& A_{\mathrm{S}}^{0}(I=2)=\frac{1}{64 \pi} \frac{m_{\mathrm{H}}^{2}}{m_{\mathrm{W}}^{2}} g^{2}\left\{\frac{1}{\hat{s}} \ln (1+\hat{s})-1\right\} .
\end{aligned}
$$

At energies much smaller than $m_{\mathrm{H}}$

$$
\begin{aligned}
& A_{\mathrm{s}}^{0}(I=0) \underset{s \ll m_{\mathrm{H}}^{2}}{=} \frac{1}{64 \pi} \frac{g^{2}}{m_{\mathrm{W}}^{2}} s, \\
& A_{\mathrm{s}}^{0}(I=2) \underset{s \ll m_{\mathrm{H}}^{2}}{=}-\frac{1}{128 \pi} \frac{g^{2}}{m_{\mathrm{W}}^{2}} s,
\end{aligned}
$$

while at very high energies

$$
\begin{aligned}
& A_{s}^{0}(I=0) \underset{s \gg m_{\mathrm{H}}^{2}}{=}-\frac{5}{128 \pi} \frac{m_{\mathrm{H}}^{2}}{m_{\mathrm{W}}^{2}} g^{2}, \\
& A_{\mathrm{s}}^{0}(I=2) \underset{s \gg m_{\mathrm{H}}^{2}}{=}-\frac{1}{64 \pi} \frac{m_{\mathrm{H}}^{2}}{m_{\mathrm{W}}^{2}} g^{2} .
\end{aligned}
$$


We see thus that the isoscalar interaction is attractive at all energies provided the Higgs particle is extremely massive or there is no scalar particle at all. This strongly suggests the formation of an $I=0$ scalar bound state, consisting of massive $\mathrm{W}_{\ell}^{+} \mathrm{W}_{\ell}^{-}$ and $/$ or $Z_{\ell} Z_{\ell}$ pairs. Although the sign of the isotensor amplitude is always negative, higher lying $I=2$ bound states are nevertheless not completely excluded. But isoscalar are highly favored over isotensor scalar bound states (compare also the coefficients in (9)-(12)).

We now want to entertain the possibility that the strong Higgs force can be described by a confining attractive potential of the form

$$
V \propto \frac{\left(s_{1} \cdot r_{12}\right)^{2}\left(s_{2} \cdot r_{12}\right)^{2}}{r_{12}^{n}} .
$$

Note that this potential in classical mechanics corresponds to a system of two particles oscillating around their center-of-mass on a DNA-like double helix. Potential (13) is proportional to the product of the spin components of the two vector particles along their relative direction, because a strong weak interaction only takes place between longitudinally polarized vector bosons. By virtue of the infrared freedom of the Higgs self-coupling, we assume furthermore that this potential is singular in case of a heavy or no Higgs particle. In a non-relativistic context this means $n \geqslant 6$, whereas it amounts to $n \geqslant 8$ in a relativistic framework (the reason being that the Schrödinger and Bethe-Salpeter equations are second- and fourth-order differential equations respectively). But the Schrödinger equation is not very appropriate here: first, the masses of the two particles are identical and secondly, a discrete spectrum of bound states forms, however, without ground state (since the hamiltonian is unbounded from below).

Solving the relativistic Bethe-Salpeter equation for two massive spin-1 particles with potential (13) is extremely hard. Let us therefore simplify the problem by replacing the vector bosons by their Higgs-Kibble ghosts (although this is probably a poor approximation if there turn out to be low lying bound states) and by considering a ladder approximation in which Higgs particles are exchanged one by one and two by two (depicted in fig. 2), corresponding to a singular potential.

The Bethe-Salpeter equation in the center-of-mass system in four-dimensional euclidean space then reads

$$
\left[\left(\square+\frac{1}{4} M^{2}-1\right)^{2}+M^{2} \frac{\partial^{2}}{\partial x_{4}^{2}}-V(r)\right] \psi(x)=0,
$$

with $M$ the bound state mass and $V$ the attractive potential

$$
V(r)=c_{1} \Delta_{\mathrm{H}}\left(r, m_{\mathrm{H}}\right)+c_{2} \Delta_{\mathrm{H}}^{2}\left(r, m_{\mathrm{H}}\right)
$$


$\Delta_{\mathrm{H}}$ denotes the causal Higgs propagator and $c_{1}$ and $c_{2}$ are two positive constants. Remark that the behavior of the wave function at small distances is completely independent of $M$ and the potential near the origin also does not depend on $m_{\mathrm{H}}$ (the fact that we are dealing with a very heavy Higgs particle is expressed by the use of a singular potential). We therefore focus on the following equation:

$$
\left[(\square-1)^{2}-\left(\frac{c_{1}}{r^{2}}+\frac{c_{2}}{r^{4}}\right)\right] \psi(x)=0,
$$

and quantize a coupling constant instead of the binding energy. Introducing polar coordinates and decomposing $\psi$ according to

$$
\psi(x)=\sum_{k=0}^{\infty} \sum_{l=0}^{k} \sum_{m=-l}^{l} \phi_{k}(r) Y_{k, l, m}\left(\theta_{1}, \theta_{2}, \theta_{3}\right),
$$

with

$$
Y_{k, l, m}\left(\theta_{1}, \theta_{2}, \theta_{3}\right) \propto C_{k-1}^{l+1}\left(\cos \theta_{1}\right)\left(\sin \theta_{1}\right)^{\prime} P_{l}^{m}\left(\cos \theta_{2}\right) \mathrm{e}^{i m \theta_{3}}
$$

the four-dimensional spherical harmonic functions, we arrive at the radial equation for the $k$-angular momentum wave function

$$
\left[\left(K_{k+1}(r)-1\right)^{2}-\left(\frac{c_{1}}{r^{2}}+\frac{c_{2}}{r^{4}}\right)\right] \psi_{k}(r)=0,
$$

with $\psi_{k}(r)=r \phi_{k}(r)$ and

$$
K_{j}(r)=\frac{\mathrm{d}^{2}}{\mathrm{~d} r^{2}}+\frac{1}{r} \frac{\mathrm{d}}{\mathrm{d} r}-\frac{j^{2}}{r^{2}}
$$

It can now be verified that both the differential equation

$$
\left[K_{k+1}^{2}(r)-\frac{c_{2}}{r^{4}}\right] \psi_{k}(r)=0,
$$

which governs the behavior of (19) at small distances, and

$$
\left[K_{v}^{u}(r) K_{v}^{-u}(r)+4 \frac{u(u-1)}{r^{2}}\right] \psi_{k}(r)=0,
$$

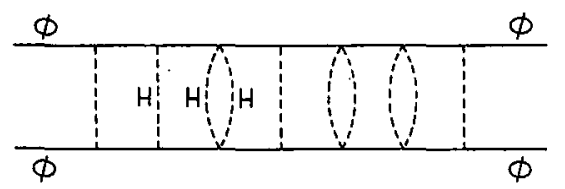

Fig. 2. Ladder approximation under consideration. 
with

$$
K_{q}^{p}(r)=r^{-p} K_{q}(r) r^{p}
$$

where

$$
\begin{aligned}
& s=k^{2}+2 k+2, \\
& t=\sqrt{k^{2}(k+2)^{2}-c_{2}}, \\
& u=1-\frac{1}{2} \sqrt{2(s-t)}, \\
& v=\sqrt{\frac{1}{2}(s+t)}
\end{aligned}
$$

(we suppose $0 \leqslant c_{2} \leqslant k^{2}(k+2)^{2}$ ) have the same acceptable power solutions. The singular part of the potential has been absorbed, up to a regular $1 / r^{2}$ term, into a slightly modified kinetic operator. We therefore replace eq. (19) by the regular

$$
\left[\left(K_{v}^{u}(r)-1\right)\left(K_{v}^{-u}(r)-1\right)+\frac{4 u(u-1)-c_{1}}{r^{2}}\right] \psi_{k}(r)=0
$$

and solve it by standard methods, yielding

$$
c_{1}=4(v+n)(v+n+1), \quad n=0,1,2, \ldots,
$$

and a corresponding spectrum of bound states with a well-defined ground state (and excitations).

It should be stressed that the very strong binding force of the (iso)scalar bound states in the absence of a Higgs particle is nothing but that part of the Yang-Mills gauge interaction, which is responsible for its bad small-distance or high-energy behavior. No new force is required. Not at all. It is now very tempting to speculate that these bound states in fact replace the Higgs: the non-renormalizable standard model without a scalar particle, being the fundamental theory, would look exactly the same as the renormalizable standard model with a Higgs particle (the latter would serve only as an effective theory). We finally want to emphasize that bound states very efficiently circumvent the screening theorem. Completely new objects and phenomena would make their appearance at relatively low energies. It has been conjectured recently that they are in fact already experimentally observed [9].

The author would like to thank Professor M. Veltman for stimulating discussions and continuous encouragement. 


\section{References}

[1] M. Veltman, Phys. Rev. Lett. 34 (1975) 777

[2] M. Veltman, Acta Phys. Pol. B8 (1977) 475;

T. Appelquist and R. Shankar, Nucl. Phys. B158 (1979) 317;

T. Appelquist and C. Bernard, Phys. Rev. D22 (1980) 200;

A.C. Longhitano, Phys. Rev. D22 (1980) 1166;

M.B. Einhorn, Nucl. Phys. B246 (1984) 75

[3] D.A. Dicus and V.S. Mathur, Phys. Rev. D7 (1973) 3111

[4] B.W. Lee, C. Quigg and H.B. Thacker, Phys. Rev. D16 (1977) 1519

[5] H.A. Gordon et al., in Proc. 1982 DPF Summer Study on Elementary particle physics and future facilities (Snowmass, Colorado, 1982)

[6] G.L. Kane, University of Michigan preprint UM TH 83-25 (1983)

[7] $M$. Veltman, in ref. [2]

[8] M. Lemoine and M. Veltman, Nucl. Phys. B164 (1980) 445;

R. Philippe, Phys. Rev. D26 (1982) 1588

[9] M. Veltman, Phys. Lett. 139B (1984) 307 